\title{
Der Zerfall des Schriftdeutschen im Estado Novo?
}

\author{
The Decay of Written German in the Vargas Era? \\ http://dx.doi.org/10.11606/1982-8837213387
}

\section{Lisa Woytowicz ${ }^{1}$}

\begin{abstract}
This essay deals with the use of written German in Brazil in the $20^{\text {th }}$ century. The basis of this research project is a collection of private letters written by Hunsruickisch speakers during the Vargas Era. The purpose of this study is to identify to what extent Hunsruickisch speakers used a single written standard, since their mother tongue was mainly spoken. It is argued that the language policy introduced by Vargas is not the only reason for Portuguese replacing German as umbrella language. Nevertheless, the Estado Novo was a decisive factor which prevented the establishment of a Brazilian variety of written standard German.
\end{abstract}

Keywords: Hunsrückisch, Linguistic Contact, German Minorities, Brazil, Vargas Era

Zusammenfassung: Der folgende Beitrag beschäftigt sich mit Sprechern des Hunsrückischen in Brasilien und deren Gebrauch des Schriftdeutschen im 20. Jahrhundert. Basis der Analyse ist ein Korpus von Privatbriefen, die während der ersten Diktatur geschrieben wurden. Der Fokus der Analyse ist die Frage nach einer uniformen Schriftnorm für diese Sprechergruppe, deren Muttersprache fast ausschließlich mündlich verwendet wurde. Es wird argumentiert, dass der Estado Novo nicht die einzige Ursache für den Dachsprachenwechsel vom Deutschen zum Portugiesischen war, jedoch entscheidend dazu beigetragen hat, dass die Etablierung einer brasilianischen Schriftnorm des Deutschen endgültig verhindert wurde.

Stichwörter: Hunsrückisch, deutsche Minderheiten, Sprachkontakt, Brasilien, Estado Novo

Resumo: Neste artigo discute-se o uso do alemão escrito por falantes da variedade hunsriqueano no Brasil no século XX. A base da pesquisa apresentada é um acervo de cartas privadas escritas na época do Estado Novo. O foco da análise é a questão de um único padrão do alemão escrito usado pelos hunsriqueanos cuja língua materna principalmente era uma variedade falada. É de supor que a política linguística na ditadura não foi o único fator que resultou na mudança da língua teto a favor do português. Mesmo assim, o Estado Novo desempenhou um papel significante porque definitivamente impediu o estabelecimento de um padrão brasileiro do alemão escrito.

Palavras-chave: hunsriqueano, minorias alemãs, contato linguístico, Brasil, Estado Novo

\footnotetext{
1 Johannes Gutenberg-Universität Mainz, Saarstraße 21, 55122 Mainz, Alemanha, email: LisaWoytowicz@gmx.de
} 


\section{Historische und linguistische Kontextualisierung}

Die ersten deutschen Auswanderer kamen im Jahr 1824 nach Brasilien und siedelten zuerst in São Leopoldo im heutigen Bundesstaat Rio Grande do Sul, ab 1829 auch im heutigen Bundesstaat Santa Catarina (vgl. Altenhofen 1996: 24; Altenhofen 2016: 111). In den folgenden Jahrzehnten entfernten sich einige ihrer Nachfahren immer weiter von der Mutterkolonie São Leopoldo. Die durch AltEnhOFEN (2014: 90) rekonstruierten Migrationsströme zeigen eine Ausbreitung der ,Deitschen““2 in weiteren Regionen Brasiliens, wodurch sich auch die von ihnen gesprochenen Varietäten des Deutschen anderorts verbreiteten.

Der vorliegende Artikel wird sich mit dem Gebrauch des Schriftdeutschen der Nachfahren dieser Einwanderer auseinandersetzen. Hierzu wurden ausgewählte Privatbriefe aus dem 20. Jahrhundert analysiert. Diese werden zuvor historisch und linguistisch eingeordnet; anschließend werden in mehreren Briefen vorkommende Phänomene aufgezeigt und diese anhand von Beispielen präsentiert. Dabei soll die Frage nach einem möglichen Zerfall des Schriftdeutschen beantwortet werden.

Der Begriff „Hochdeutsch“ wird in dieser Arbeit „,im sprachsoziologischen Sinne [als] die (überregional gültige, normierte, kodifizierte) Hochsprache“ (BUßMANN 2008: 265) verstanden. BußMAnN (2008: 680) setzt die Begriffe Hochsprache und Standardsprache miteinander gleich. Beide bezeichnen „die historisch legitimierte, überregionale mündliche und schriftliche Sprachform der sozialen Mittel- bzw. Oberschicht“ und unterliegen „,weitgehender Normierung, die über öffentliche Medien und Institutionen, vor allem aber durch das Bildungssystem kontrolliert und vermittelt [werden]“ (ebd.). Hochdeutsch entspricht daher Standarddeutsch. In der vorliegenden Arbeit wird jedoch nur erstgenannter Begriff verwendet. Schriftdeutsch ${ }^{3}$ ist wiederum „das Hochdeutsche in der (bestimmten sprachlichen Gesetzmäßigkeiten folgenden) schriftlichen Form“ (DUDENREAKTION 2017).

\footnotetext{
${ }^{2}$ Der Begriff verweist auf Deutschbrasilianer, d.h. Einwanderer der ersten Migrationswelle von 18241850, im Gegensatz zu „Deutschländern“, die erst später nach Brasilien auswanderten (vgl. ALTENHOFEN 2016: 111).

${ }^{3}$ In der Schweiz werden die Begriffe Hochdeutsch und Schriftdeutsch gleichgesetzt. In dieser Arbeit werden die beiden Begriffe im oben definierten Sinne voneinander differenziert (vgl. DUDEN 2017).
} 
Woytowicz, L. Zerfall des Schriftdeutschen im Estado Novo?

Für die Einwanderer der ersten Generationen fungierte das Hunsrückische als lingua franca in informellen Kontexten, d.h. insbesondere in der gesprochenen Sprache. Die Einwanderer kamen aus unterschiedlichen Regionen des damaligen Deutschen Bunds und sprachen daher unterschiedliche Varietäten. Hunsrückisch bildete sich aufgrund seiner Nähe zum Hochdeutschen als Koiné zur gegenseitigen Verständigung innerhalb dieser heterogenen Gruppe heraus. Im Gegensatz dazu fungierte das Hochdeutsche als Dachsprache im formalen, ergo meist geschriebenen, Kontext (vgl. ALTENHOFEN 2014: 86 f.). Der Gebrauch des Hochdeutschen bzw. des Hunsrückischen war somit diaphasisch markiert.

„In die neu entstandene Koiné wurden nach und nach immer mehr portugiesische Elemente übernommen, sodass sie zu einer eigenständigen Varietät wurde, für die eine interne Variation charakteristisch ist“ (STEFFEN 2016: 135). ALTENHOFEN definiert das Hunsrückische im Gegensatz zum Hochdeutschen wie folgt:

»Hrs.« ist der Oberbegriff für eine überregionale Varietät des Deutschen in Rio Grande do Sul/Südbrasilien, die ein Dialektkontinuum darstellt, dessen sprachliche Konstitution auf eine rhein-/moselfränkische Basis zurückgeht und eine Vielfalt sprachkontaktbedingter Elemente anderer deutscher Dialekte sowie insbesondere des Ptg. einschließt (ALTENHOFEN 1996: 27).

In den sogenannten deutschen Kolonien entstanden selbst verwaltete Schulen, an denen (das lokale) Hochdeutsch gelehrt wurde. Es „nahm daher naturgemäß die Rolle der normgerechten Schriftsprache ein“ (PUPP SPINASSÉ 2016: 88). Außerdem erschienen vor allem im Umkreis der Mutterkolonie zahlreiche Printmedien auf Deutsch (vgl. ALTENHOFEN 2016: 125; STEFFEN 2016: 136). ALTENHOFEN (2016: 104) grenzt diese Form des Hochdeutschen als „landschaftliches Hochdeutsch“ ab. Darunter versteht er „die lokal intendierte Oralisierungsnorm des Deutschen, die aufgrund [linguistischer] Merkmale, von diesen Sprechern als gehobene Normsprache bewertet wird, die sie jedoch mit einem »Regionalakzent« realisieren und vorwiegend mit partieller Kompetenz beherrschen“. Es ist daher davon auszugehen, dass weite Teile der ersten Generationen der Einwanderer zwei verschiedene Varietäten des Deutschen benutzten, Portugiesisch hingegen kaum beherrschten.

Der Begriff „landschaftliches Hochdeutsch“ ergibt insbesondere mit Verweis auf die oben genannte Definition Sinn, der zufolge v.a. das Bildungssystem den sprachlichen Standard vermittelt und kontrolliert. Die Deutungshoheit darüber, was Hochdeutsch in Brasilien zu dieser Zeit war, lag bei den Schulen in den deutschen 
Kolonien. Diese vermittelten zwangsläufig ein alternatives Hochdeutsch, da der Formierungs- und Konsolidierungsprozess des europäischen Hochdeutsch zum Zeitpunkt der ersten deutschen Auswanderungswelle nach Brasilien noch gar nicht abgeschlossen war (vgl. FLEISCHER et. al. 2001: 635 ff.). Das Schriftdeutsch von Hunsrückischsprechern basierte daher auf dem landschaftlichen Hochdeutsch, das sich anders als sein europäisches Pendant entwickelte.

Die in diesem Beitrag vorgestellten Briefe sind ein Bruchteil der Forschungsarbeit, die im Rahmen des Projekts „ALMA-H“ (Atlas LinguísticoContatual das Minorias Alemãs na Bacia do Prata - Hunsrückisch, auf Deutsch: Atlas zum Sprachkontakt der deutschen Minderheiten im Rio-de-la-Plata-Becken Hunsrückisch) geleistet wird. ${ }^{4}$ Sie sind Bestandteil einer privaten Sammlung von etwa 1200 Briefen, die von deutschen Siedlern in Brasilien und deren Nachfahren im 19. und 20. Jahrhundert verfasst wurden. Daher wurden sie teilweise in Deutscher Kurrentschrift (auch Spitzschrift) verfasst und mussten vor ihrer Analyse transkribiert werden.

STEFFEN (2016: 138 ff.) definiert anhand einer Auswahl von Briefen aus demselben Korpus verschiedene Phasen des Sprachkontakts. In der ersten Phase, die ungefähr bis zur Jahrhundertwende andauert, lassen sich vereinzelt Lusismen in den Briefen nachweisen. Die meist lexikalischen Entlehnungen beschränken sich auf bestimmte Wortfelder und brasilianische Realia. In der Regel werden die Lehnwörter stark assimiliert. STEFFEN formuliert - basierend auf der Analyse mehrerer Briefe aus demselben Zeitraum - in Bezug auf die Schreibung der Entlehnungen drei Gesetzmäßigkeiten:

1. Sie entspricht in der Regel nicht der portugiesischen Orthographie; 2. Es werden häufig Aussprachemuster des hunsrückischen Dialekts in der Schreibung widergespiegelt; [...] 3. Die Schreibung weist eine große Varianz auf, was sich mitunter auch auf den gleichen Schreiber beziehen kann. (STEFFEN 2016: 142)

Mit Anbruch des 20. Jahrhunderts werden erste Briefe vollständig auf Portugiesisch verfasst. Häufig kommt es auch innerhalb eines Briefes zu Codeswitching. Bis zum Zweiten Weltkrieg überwiegt bei den meisten Autoren allerdings das Deutsche. Diese zweite Phase ist STEFFEN zufolge im Gegensatz zur ersten von der Zweisprachigkeit

\footnotetext{
${ }^{4}$ Dabei handelt es sich um ein Gemeinschaftsprojekt der Romanistik der Christian-Albrechts-Universität Kiel und der Germanistik des Instituto de Letras der Universidade Federal do Rio Grande do Sul, Brasilien. Siehe auch: http://www.ufrgs.br/projalma.
} 
vieler Einwanderer und ihrer Nachfahren geprägt (vgl. 2016: 143 ff.). Jedoch sei „die eigene Umgangsvarietät schon stark gemischt“ (STEFFEN 2016: 147). Dies zeigt sich u.a. anhand von Kasusverlust bzw. -unsicherheit, Lehnwörtern aus zusätzlichen Wortfeldern und einer „portugiesischeren“ Schreibweise der Entlehnungen. Parallel kommt es sowohl im geschriebenen Portugiesisch als auch im geschriebenen Hochdeutsch (weiterhin) zu phonetischen Interferenzen aus dem Hunsrückischen. Angesichts des Jonglierens mit drei Varietäten könnte man daher sogar von einer Dreisprachigkeit der Autoren der Briefe sprechen. Nichtsdestotrotz ist nicht jede Abweichung vom Hochdeutschen, das in der geschriebenen Sprache nach wie vor als normgerecht galt, auf einen Einfluss des Portugiesischen oder des Hunsrückischen zurückzuführen (vgl. STEFFEN 2016: 147 ff.). STEFFEN (2016: 150) betrachtet die oben benannten linguistischen Phänomene auch als Resultat einer „Herausbildung einer eigenständigen deutschbrasilianischen Identität ${ }^{\star 5}$. Dabei beruft er sich auf ROSENBERG:

In Zeiten ernsthafter Norminstabilität kann es zu "galoppierendem" Wandel kommen: Die Normloyalität geht zurück, wenn kulturelle und sprachliche Gruppengrenzen undeutlich werden. Die Normsicherheit kann rapide zerfallen unter intensivem Sprachkontakt, der die Herausbildung von Mischvarietäten oder sogar Sprachmischung bewirkt [...] (ROSENBERG 2003: 14).

Einer der Faktoren, die kulturelle und sprachliche Gruppengrenzen im Brasilien des 20. Jahrhunderts ins Wanken brachten, war die (Sprach-)Politik im sogenannten Estado Novo (1937-1945) unter Getúlio Vargas. Im Zuge des Nationalisierungsprozesses wurde jeglicher Gebrauch einer anderen Sprache als Portugiesisch untersagt. Dies war einer der hauptsächlichen Gründe für den von ALtENHOFEN (2016: 124) beschriebenen Dachsprachenwechsel vom Hochdeutschen zum Portugiesischen, „der sich in der diagenerationellen Dimension vollzieht". Einerseits beschleunigte diese Politik den Verlust des Hochdeutschen, indem Deutsch als Schulfach oder als Publikationssprache verboten wurde, andererseits fehlte es an Infrastruktur für das Erlernen des Portugiesischen als Fremdsprache (vgl. ebd. 124 ff.; STEFFEN 2016: 133 ff.; PuPP SPINASSÉ 2016: 88).

In der dritten von STEFFEN (2016: 150 ff.) beschriebenen Phase, deren Anfang das Ende des Zweiten Weltkriegs und der Fall des Vargas-Regimes bilden, verblasst das Deutsche in den Briefen zusehends. Die Normloyalität nimmt weiter ab, jüngere

\footnotetext{
${ }^{5}$ Dies ist wiederum eine der Grundvoraussetzungen für die Entstehung eigener Varietäten (vgl. FLEISCHER et. al. 2001: 360).
} 
Autoren können häufig keine vollständigen Texte mehr auf Hochdeutsch formulieren und verfallen ins Portugiesische, das sie jedoch ebenfalls nicht einwandfrei beherrschen. Im Gegensatz zu der Generation, die Deutsch in der Schule gelernt hatte, konnten die ersten Betroffenen des Dachsprachenwechsels sich somit schriftlich weder auf Deutsch noch auf Portugiesisch normgerecht ausdrücken.

STEFFEN (2016: 155) kommt zu dem Schluss, dass der Estado Novo keinen direkten Einfluss auf die private Schriftlichkeit der Hunsrückischsprechenden nahm, da die Normloyalität bereits um die Jahrhundertwende abgenommen hatte. Vielmehr sei der „Sprachwechsel im Bereich der Privatschriftlichkeit [...] ein kontinuierlicher, aber sehr langsam ablaufender Prozess, der durch das allmähliche Ein- und Vordringen des Portugiesischen bei gleichzeitigem Abbau des Hochdeutschen geprägt war".

RosenBerg (2003: 9) zufolge setzte in den Kolonien mit dem Estado Novo eine „Redialektalisierung“ ein; STEFFEN (vgl. 2016: 133) ist der Meinung, dass dieser Prozess den Dachsprachenwechsel vorantrieb. Man könnte aber auch davon ausgehen, dass der durch zahlreiche Faktoren - wie Sprachpolitik, Urbanisierung und Industrialisierung (vgl. ALTENHOFEN 1996: 74) - voranschreitende und politisch gewollte Dachsprachenwechsel die Redialektalisierung förderte. Diese Annahme geht Hand in Hand mit Altenhofens (2016: 126) fester Überzeugung, dass „die Nationalisierung im Wesentlichen den Zugang zur hochdeutschen Dachsprache verdrängt hat, [aber] nicht in gleichem Maße [...] das Hunsrückische, das [...] eine Art sprachliches Refugium bildete“. Eine „Flucht“ ins Hunsrückische, der einzigen Muttersprache, war daher nur eine logische Konsequenz aus dem fehlenden Zugang zu Deutsch- und angemessenem Portugiesischunterricht zu dieser Zeit. Es kann überdies als ein „Sich-Berufen“ auf die eigene Identität interpretiert werden; wie bewusst bzw. unbewusst dieser Prozess sich vollzog, ist jedoch kaum zu ermessen.

Die Autorin vertritt die These, dass es sich (1) beim Hunsrückischen um die einzige Muttersprache ${ }^{6}$ seiner Sprecher handelt und dass (2) diese Varietät für ihre Sprecher schon immer die Rolle des sprachlichen, identitätsprägenden Refugiums eingenommen hat; im Estado Novo, einer Zeit, in der „kulturelle und sprachliche Gruppengrenzen undeutlich [wurden]“" (ROSENBERG 2003: 14), wurde diese Rolle

\footnotetext{
6 „Einzige Muttersprache“ meint, dass es sich beim Hunsrückischen um die einzige Varietät handelte, die von den Sprechern auf muttersprachlichem Niveau beherrscht wurde, da sie diese bereits als Kind vor Eintritt in die Schule lernten und sich in dieser Sprache am sichersten und wohlsten fühlten.
} 
Woytowicz, L. Zerfall des Schriftdeutschen im Estado Novo?

besonders gestärkt. Für diese Behauptungen spricht u.a. die Tatsache, dass schon zu Anfang des 20. Jahrhunderts vereinzelt Texte auf Hunsrückisch verfasst wurden; diese hatten i.d.R. humoristische oder identitäre Zwecke (vgl. PUPP SPINASSÉ 2016: 88; AltenHOFEN 2016: 112; AltenHOFEN et al. 2007: 73 f.). Außerdem schrieben spätere Generationen, denen der Hochdeutschunterricht in der Schule verwehrt blieb, in Anlehnung an die ersten Autoren ebenfalls „so wie sie sprachen“, ergo auf Hunsrückisch (PUPP SPINASSÉ 2016: 88), ohne dass die Varietät zuvor schriftlich genormt worden war.

Dies - verstärkt durch den Willen, in der Sprache schreiben zu können, in der man sich am wohlsten fühlt - erzeugte eine (kleine, aber immerhin vorhandene) Schreibtradition im Hunsrückischen [...]. Basierend auf dieser Tradition wurden im Rahmen des Projekts ESCRITHU Schriftregeln für das Hunsrückische erstellt, die vom Standarddeutschen ausgehend die Entscheidungen der alten Autoren/Sprecher und die Variationen innerhalb des Hunsrückischen berücksichtigen [...] (PUPP SPINASSÉ 2016: 88, Hervorh. d. Verf.).

Es scheint, als ob den Sprechern aller Generationen das Hunsrückische allgemein näher war bzw. ist als das Hochdeutsche oder das Portugiesische. Jedoch wurde aufgrund politischer, gesellschaftlicher und historischer Aspekte, erst dem Hochdeutschen und später dem Portugiesischen in der Schriftsprache stets mehr Bedeutung und daher mehr Prestige beigemessen. Aus diesem Grunde gab es auch erst im 21. Jahrhundert erste Ansätze, Schriftregeln für das Hunsrückische festzulegen. Der Prestigeunterschied erklärt außerdem, warum Verfasser sich in älteren schriftlichen Belegen, die zahlreiche Merkmale des Hunsrückischen aufweisen, trotzdem oft an dem orientieren, was sie für Hochdeutsch halten (vgl. AltenHOFEN 2016: 112 f.), obwohl sie diese Varietät „vorwiegend mit partieller Kompetenz beherrschen“ (ebd. 2016: 104; vgl. ALTENHOFEN et al. 2007: 74). Es scheint daher abwegig, Hochdeutsch als ihre Muttersprache zu bezeichnen.

\section{Eine Auswahl an Briefen: allgemeine Beobachtungen}

Die im Folgenden vorgestellten Briefe wurden im 20. Jahrhundert verfasst und an eine Frau namens Ida Werner bzw. ihre Familie in Montenegro geschickt. Heute befinden sich die Briefe im Besitz ihrer Nachfahren in Rolante (siehe Karte 1). Die Empfängerin der Briefe lebte somit im näheren Umkreis der Mutterkolonie São Leopoldo, in der 
Woytowicz, L. Zerfall des Schriftdeutschen im Estado Novo?

Deutsch lange Zeit präsenter war als in den später gegründeten Tochterkolonien, da dieses Gebiet räumlich, sozial und sprachlich bis in die 1970er-Jahre relativ isoliert blieb (vgl. Altenhofen 2016: 110 f.). Die Absender der Briefe sind Freunde und Verwandte der Empfängerin. Einige wohnten ebenfalls im näheren Umkreis der Mutterkolonie, in Canoas, Novo Hamburgo und Rolante; andere hatten sich im Zuge der Binnenmigrationsströme weiter von São Leopoldo entfernt und lebten in Santa Rosa oder Curitiba (siehe Karte 2).

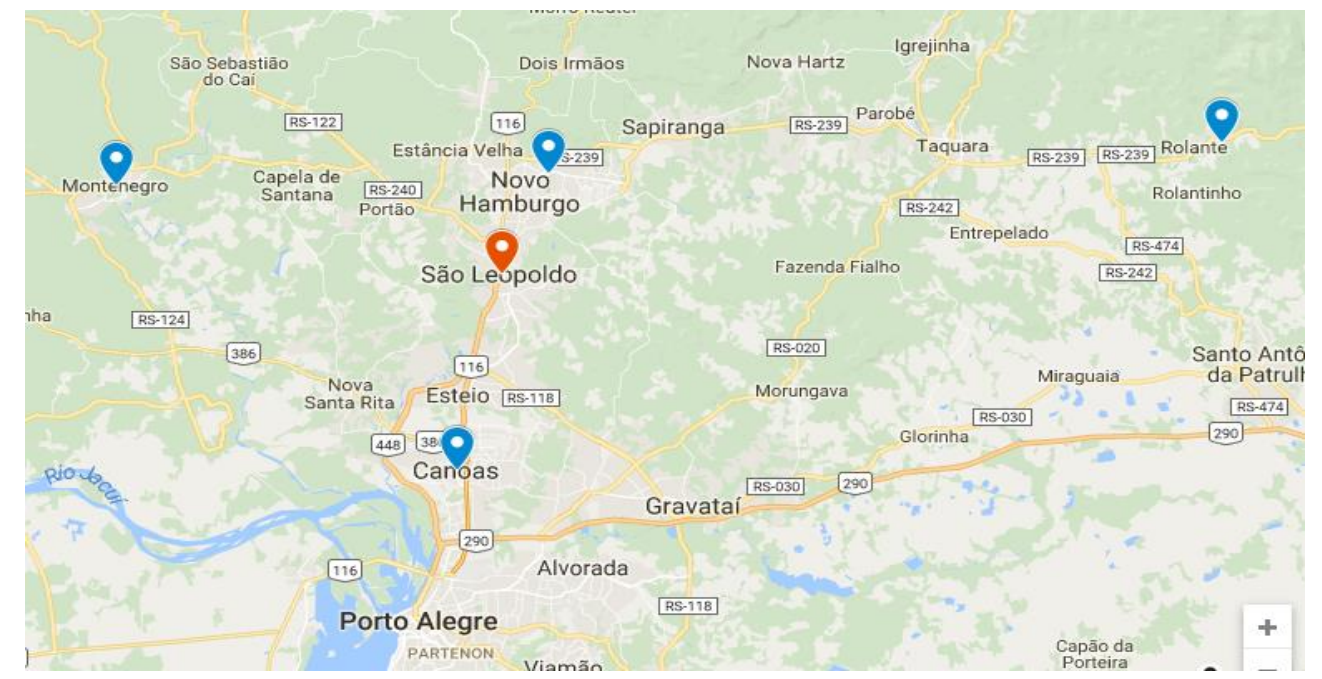

Karte 1: Wohnort der Empfängerin und der Absender aus dem näheren Umkreis (GoOGLE: 2017)

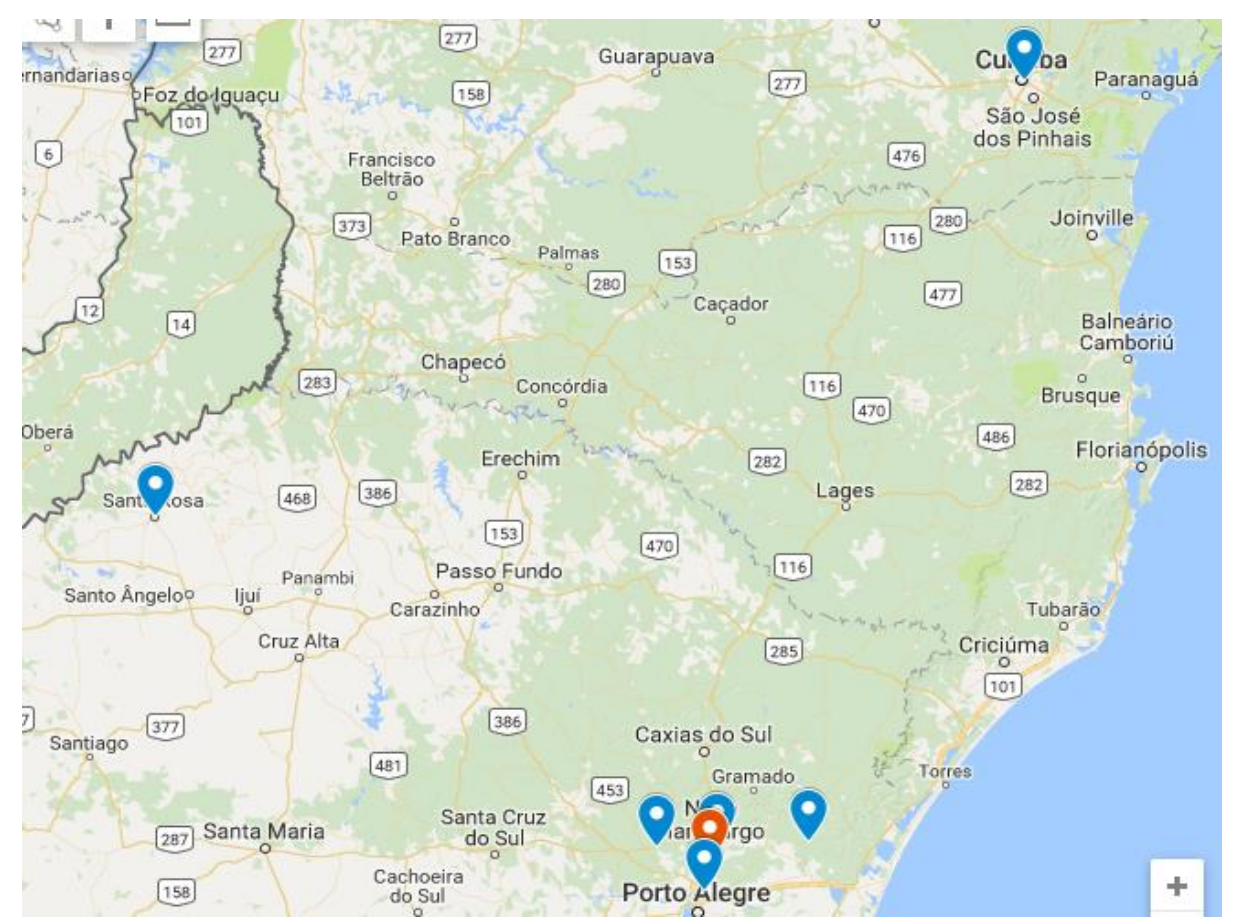

Karte 2: Wohnorte der Absender aus dem weiteren Umkreis (GOOGLE: 2017) 
Inhaltlich geht es in den meisten Briefen in erster Linie um den Alltag der Verfasser. Oft schreiben sie zu bestimmten Gelegenheiten wie Geburtstagen oder an christlichen Feiertagen wie Pfingsten, Ostern oder Weihnachten. Alle in diesem Beitrag präsentierten Briefe wurden auf Deutsch verfasst und beinhalten kein Codeswitching im klassischen Sinne; der Kodewechsel erfolgt nie über längere Passagen. Es kommt jedoch zu Entlehnungen, nonce words und Ein-Wort-Switches. ${ }^{7}$ Portugiesischsprachige Briefe, deren Anzahl im 20. Jahrhundert und insbesondere im Estado Novo anstieg, wurden für diesen Beitrag bewusst ausgeklammert. Fokus der Analyse sind die Briefe, die während oder unmittelbar vor der Diktatur auf Deutsch geschrieben wurden.

Allgemein lassen sich nachstehende Aussagen über die Briefe treffen; teilweise stimmen sie mit dem überein, was STEFFEN bereits in Bezug auf die Briefe der ersten Einwanderergenerationen und die zweisprachigen Briefe des 20. Jahrhunderts festgestellt hat. (1) Die Briefe weisen einen hohen Grad an Mündlichkeit auf. STEFFEN (2016: 138) begründet dies damit, dass Privatbriefe dem kommunikativen Nähepol besonders nahe stünden, d.h. dass diese Briefe konzeptionell mündlich sind und über einen geringen Planungsgrad verfügen. (2) Dies bedeutet konkret, dass „,die Texte in der Regel nicht in reinem Hochdeutsch geschrieben sind, sondern sowohl dialektale Elemente des riograndenser Hunsrückisch enthalten als auch lusische Elemente“ (ebd.). Der hohe Grad an Mündlichkeit äußert sich u.a. in fehlender Interpunktion, Wiederholungen bzw. Redundanzen.

Kein einziger der Briefe entspricht dem Hochdeutschen; die Verfasser orientierten sich beim Schreiben an dem von ALTENHOFEN (2016: 104) definierten „landschaftlichen Hochdeutsch“, das bis zum Verbot der Sprache an den selbst verwalteten Schulen gelehrt wurde und als die erstrebenswerte Schriftnorm galt (siehe oben). Diese Varietät enthielt bereits Elemente des Hunsrückischen, das seinerseits wiederum Elemente des Portugiesischen enthielt (vgl. STEFFEN 2016: 147; vgl. PUPP SPINASSÉ 2016: 82). Es ist daher nahezu unmöglich, eventuell auch nicht

\footnotetext{
${ }^{7}$ BRANFORD und CLAUGHTON (1995: 209) definieren Entlehnen als "the adoption into one language of items, patterns and meanings from another". Sie differenzieren klar zwischen Entlehnungen und ad hoc gebildeten Wörtern - nonce words. Letztere sind kein Teil des von den Autoren so benannten öffentlichen Sprachsystems, innerhalb dessen der Großteil der Sprecher einer bestimmten Varietät dieselben Entlehnungen akzeptiert und verwendet. Im Unterschied zu nonce words werden Ein-Wort-Switches nicht an die Zielsprache angepasst. Sie stellen keine Entlehnungen dar, sondern einen sehr kurzen Wechsel in eine andere Varietät. Im Falle der Briefe kann man daher in manchen Fällen nur vermuten, bei welchen Wörtern es sich um etablierte Entlehnungen, um nonce words oder um Ein-Wort-Switchs handelt. Daher werden alle drei im weiteren Verlauf unter „Entlehnungen“ zusammengefasst.
} 
wünschenswert, bei der Analyse der deutschen Briefe stets zwischen Einflüssen bzw. Interferenzen der unterschiedlichen Varietäten unterscheiden zu wollen; ihre Grenzen verlaufen fließend.

(3) Die meisten Schreiber sind inkonsistent in der Anwendung von (orthographischen) Regeln. Teilweise werden selbst innerhalb eines Briefes einzelne Wörter von demselben Autor unterschiedlich geschrieben. Dies wirft die Frage nach der Existenz einer oder mehrerer Standards auf. (4) Im Vergleich zum Schriftdeutschen weist das landschaftliche Hochdeutsch Anzeichen eines Abbaus grammatikalischer Strukturen auf. Es kommt u.a. zu Kasusverlust und einer unüblichen Nutzung von Pronomina (vgl. STEFFEN 2016: 147 f.).

(5) Alle Autoren der hier vorgestellten Briefe können sich schriftlich auf einer Varietät ausdrücken, die für sie Hochdeutsch repräsentiert. Das Ausmaß der unter den Punkten (1) bis (4) erwähnten Phänomene variiert allerdings sehr stark, ist also vom Individuum abhängig. STEFFENS (vgl. 2016: 142, siehe oben) Beobachtungen in Bezug auf portugiesische Entlehnungen in den Briefen des 19. Jahrhunderts gelten auch für die Briefe des 20. Jahrhunderts und auch für deutsche Wörter, wie im Folgenden dargestellt werden wird.

\section{Beispiele und Analyse}

Nachstehend werden meist zwei Briefe desselben Autors vorgestellt. Dies ermöglicht ggf. Rückschlüsse auf Entwicklungen im schriftlichen Ausdruck der betreffenden Person, wenn die Briefe aus verschiedenen Jahren stammen.

Liebe Ida

Hoffe das diese Zeilen Dir bei gesundheit antreffen mögen / so vie der selbe fall bei mir auch ist. Nun waramm varum / varst Du nicht gekommen für die austellung? es hatt mir / sehr leid getan denn dein Verehrer hatte auf dich gevartet / ich var zveimal an die / Station gegangen, ich hatte gemeint / ich müsste Dich bei guken. Ein paar Tage noch vordem / hatte Papa die gelegenheit mit F derüber zu sprechen / F hatte gesagt vas hatt das leben noch für einen / Zweck für mich keine Frau, da sagte Papa zu Ihm / da vere ein Mädel die Sie schon oft gesehen hat / und Sie ham Ihr gefallen Sie hatt gefragt ob Sie / noch hier veren, da ist Er ganz dumm gevorden / ganz ausersich gevorden vor / Freude er ist so froh. / [...] ich veis es Er tete zu gerne hairaten / Du Mir ist es als das noch dein richtiger Deckel vere. / Nechsten Monat den 15ten gibst vieder am Denk= / mal fest, jetzt komme aber sicher venn Du / konnst komme F den 14ten. das du am 15ten schon hier / bist ganz sicher vird 3 tage das fest sein. Freitag Samstag / und Sonntag aber ich bin ich bitte Dich nochmals / komme. So ein Almofadinha virst Du doch nicht / entgehen lassen. Est tete mich so freuen das venn / ich hairate das denn ${ }^{\text {Du }}$ 
ae auch hairaten tetest. Für / heute muss ich schliesen Mit Gruss deine / J. komme den 14 ten

[J., Hamburgo Welho, 24/10/1929]

Auf den ersten Blick fallen die zahlreichen Korrekturen auf. Häufig wurden Wörter durchgestrichen oder im Nachhinein hochgestellt hinzugefügt. Dies könnte als Unsicherheit der Autorin interpretiert werden; sicherlich ist es jedoch ein Indikator dafür, dass sie sich an einem Standard orientiert, den sie wahrscheinlich in der Schule gelernt hat. Wäre es nicht ihre Intention, normgerecht zu schreiben, hätte sie wohl kaum das Bedürfnis, sich zu korrigieren. Dieser Rückschluss kann auch anhand anderer Beobachtungen gezogen werden: Substantive werden manchmal groß (,Verehrer“, „Zweck“, „Freude“) und manchmal klein geschrieben (,gesundheit“, „fall“, „ausstellung“, „fest“"). Der Autorin scheint jedoch bewusst zu sein, dass Substantive allgemein großgeschrieben werden, denn ausgerechnet die beiden einzigen lexikalischen Entlehnungen aus dem Portugiesischen, bei denen es sich um Substantive handelt, werden am Anfang mit einem Großbuchstaben versehen: „Station“ (estação = Bahnhof) und „Almofadinha“ (almofadinha = (umgangssprachlich) gutgekleidete, reiche Person). Vermutlich sollen die Nomina so eingedeutscht werden. Allgemein scheint die Kleinschreibung von Substantiven entweder zufällig oder aufgrund von Nachlässigkeit zu erfolgen. So werden sowohl Abstrakta als auch Konkreta manchmal kleingeschrieben; das Substantiv „Tage“ wird sogar einmal groß- und einmal kleingeschrieben. Allgemein bestätigt dies ALtenHOFENS (vgl. 2016: 104, siehe oben) Aussage in Bezug auf landschaftliches Hochdeutsch.

Es bestehen jedoch auch Regelmäßigkeiten. So unterscheidet die Autorin orthographisch nie zwischen „das“ und „dass“. Dieses Phänomen lässt sich bei sehr vielen Briefen sowohl aus dem 19. als auch aus dem 20. Jahrhundert beobachten. Es mag sich dabei um eine Eigenart des landschaftlichen Hochdeutsch handeln. Dasselbe gilt für den doppelten Bindestrich bzw. das Gleichzeichen, das als Bindestrich fungiert. Der Grad der Mündlichkeit ist in diesem Brief sehr hoch: Die Autorin schreibt redundant (,vor Freude, er ist so froh"), wiederholt sich (,,jetzt komme aber sicher venn Du kommst komme [...] ich bitte Dich nochmals komme“) und bildet lange Sätze, setzt jedoch kaum Interpunktionszeichen. Besonders extrem ist dies zu beobachten, als sie ein Gespräch widergibt und erst nach acht Zeilen einen Punkt setzt. Es kann angenommen werden, dass die Autorin so schreibt, wie sie spricht. Einige Elemente 
Woytowicz, L. Zerfall des Schriftdeutschen im Estado Novo?

könnten auf das Hunsrückische zurückgeführt werden, wie z.B. vereinzelte Sonorisierung (,,ausersich“, „schliesen) oder Vokalverschiebungen („derüber“, „,vere“, „Nechste“, „tete“), die sich in der Schriftsprache der Autorin widerspiegeln. Im Vergleich zum Hochdeutschen handelt es sich dabei um Abweichungen von der Norm. $\mathrm{Ob}$ es sich in allen Fällen jedoch auch von einer Abweichung des landschaftlichen Hochdeutsches - der lokalen Norm - handelt, ist fraglich, da dieses durchaus vom Hunsrückischen geprägt war. Der hohe Grad der Mündlichkeit ist wohl kaum eine bewusste Entscheidung; es ist vielmehr ein Resultat der partiellen Kenntnisse der schriftlichen Norm (vgl. ALTENHOFEN 2016: 104) verbunden mit der kommunikativen Nähe der Briefe (vgl. STEFFEN 2016: 138). Des Weiteren muss an dieser Stelle betont werden, dass die Schulen, an denen Deutsch unterrichtet wurde, selbst verwaltet wurden und nicht selten geografisch und sozial relativ isoliert waren (siehe oben). Es wäre daher nicht besonders abwegig, von mehreren Varianten des landschaftlichen Hochdeutsch auszugehen, die an den unterschiedlichen Schulen unterrichtet wurden und in jeweils unterschiedlichem Ausmaß vom Hunsrückischen bzw. vom Portugiesischen geprägt waren.

Für das fehlende Subjekt in der ersten Zeile des Briefes bestehen mindestens zwei mögliche Erklärungen: Einerseits könnte es sich dabei um einen weiteren Indikator für Mündlichkeit handeln; andererseits kann das fehlende Subjekt mit dem Einfluss des Portugiesischen erklärt werden, da diese Sprache ohne explizites Subjektpronomen auskommt.

Abgesehen von zwei Ausnahmen wird das Graphem „w“ des Hochdeutschen

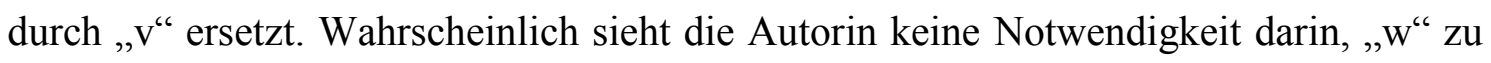
benutzen, da es phonetisch für sie keinen Unterschied macht; beide Grapheme repräsentieren anscheinend den stimmhaften labiodentalen Frikativ/v/. Da das Graphem „f“ hingegen verwendet wird, ist davon auszugehen, dass es das stimmlose Pendant /f/ repräsentiert. Die ungewöhnliche Schreibung des Ortsnamens könnte mit seinem Alter zusammenhängen. Der Ort wurde von deutschen Siedlern gegründet, die kein bis kaum Portugiesisch sprachen bzw. schrieben. Unter den Einwanderern und ihren Nachkommen setzte sich diese - im Portugiesischen falsche - Schreibart wahrscheinlich durch und wurde bis zum Nationalisierungsprozess auch nicht hinterfragt oder geändert. Ähnlich wie im Fall der fehlenden graphemischen Unterscheidung von „das“ und „dass“, trifft die fehlende Unterscheidung von /w/ und 
/v/ auf mehrere Autoren zu. Es bestätigt einmal mehr den hohen Grad der Mündlichkeit und legt die Vermutung nahe, dass es sich auch hierbei um eine Eigenart des landschaftlichen Hochdeutsch handeln könnte.

Der Abbau grammatikalischer Strukturen zeigt sich vor allem anhand der Verwendung von Personalpronomina, die abweichend vom europäischen Standard dekliniert werden (,Dir bei gesundheit antreffen mögen“) oder deren Bezüge nicht mehr deutlich sind: „,[D]a sagte Papa zu Ihm da vere ein Mädel die Sie schon oft gesehen hat und Sie ham Ihr gefallen Sie hatt gefragt ob Sie noch hier veren, da ist Er ganz..." Außerdem werden Pronomina eher zufällig großgeschrieben. Es lässt sich dabei keine zugrundeliegende Logik erkennen, da weder die Art des Pronomens (Personal- oder Possessivpronomen) noch die grammatikalische Person die Entscheidung für oder gegen einen Großbuchstaben am Anfang des Wortes beeinflusst. Meistens wird ein Personalpronomen in der zweiten Person Singular jedoch großgeschrieben; dafür spricht, dass die Autorin weiß, dass in der von ihr angestrebten Norm, Pronomina großgeschrieben werden können; anscheinend weiß sie aber nicht genau, in welchen Fällen dies zutrifft. Die Verwendung des Pronomens in der Formulierung ,ich hatte gemeint ich müsste Dich bei guken“ in Verbindung mit der Präposition „bei“ weicht deutlich vom Hochdeutschen ab; es ist jedoch möglich, dass dieser Ausdruck die lokale Norm repräsentiert. Gleiches gilt für die Konjunktivformen im letzten Absatz des Briefs; das Verb „tun“ wird im Konjunktiv II mit einem Infinitiv kombiniert.

Der zweite Brief wurde von derselben Person sechs Jahre später geschrieben. Die Autorin ist von Hamburgo Velho ${ }^{8}$ nach Porto Alegre (siehe Karte 1) gezogen. Im Gegensatz zum ersten Brief korrigiert sich die Autorin nicht mehr, was ein Argument dafür sein könnte, dass sie sich sicherer in ihrem schriftlichen Sprachgebrauch fühlt. Nach wie vor schreibt sie einige Substantive klein (,angst“, „fall“, „schvestern“) verzichtet meistens auf das Graphem „w“ und formuliert manchmal redundant (,venn ich im fall“, ,,venn, es mit der Bondes als“").

\section{Liebe Freundin.}

Ich habe Deinen Brief am 9ten erhalten. / Wenn der Dampfer ankommt, bin ich sicher / dá, brauchst keine angst zu haben, venn / ich im fall noch nicht dort sollte sein denn / varte bis ich komme, man kann nicht vissen / venn, es mit der Bondes als vass passiert dass / man sich verspétet, nun es vird schon alles / klappen. Nun verde ich schliesen

\footnotetext{
${ }^{8}$ Hamburgo Velho ist (heutzutage) ein Stadtteil von Novo Hamburgo. Auf Karte 1 ist Hamburgo Velho als blaue Markierung nördlich von Hamburgo Novo eingezeichnet.
} 


\section{Woytowicz, L. Zerfall des Schriftdeutschen im Estado Novo?}

aufs baldige / vieder sehen. Grüsse an deine liebe Mutter / und schvestern, besonders auch von deiner / Freundin J.

[J., Porto Alegre, 10/09/1935]

Weiterhin kommt es zu Vokalverschiebung („denn“) und Sonorisierung (,schliesen“). Es ist also immer noch ein hoher Grad an Mündlichkeit gegeben. Die Großschreibung von Pronomina erfolgt weiterhin eher zufällig, im Unterschied zum ersten Brief sind ihre Bezüge jedoch immer klar. Auch hier wurde die einzige lexikalische Entlehnung aus dem Portugiesischen eingedeutscht: „Bondes“ (o bonde = die Straßenbahn) wurde wie ein deutsches Nomen großgeschrieben, sein Genus wurde dem deutschen Genus (die Bahn) angepasst. Das fehlende Subjekt in der dritten Zeile kann, wie auch schon im Beispiel oben, sowohl als Interferenz der gesprochenen Sprache als auch des Portugiesischen betrachtet werden.

Es lassen sich jedoch auch Unterschiede zwischen den beiden Briefen feststellen. Die Verfasserin benutzt für das Deutsche atypische Grapheme (,dá“, „verspétet"). Durch den Umzug in eine größere Stadt war sie dem Portugiesischen im Alltag wahrscheinlich wesentlich mehr ausgesetzt als in Hamburgo Velho. Ob sie mit den diakritischen Zeichen, die Bestandteil des portugiesischen Graphemsystems sind, einen Unterschied in der Aussprache des jeweiligen Wortes kennzeichnen möchte, ist unklar. Es ist ebenso möglich, dass sie sich nicht bewusst dazu entschieden hat, die Zeichen zu verwenden. Eine weitere Auffälligkeit, die sich im ersten Brief in der Form nicht nachweisen ließ, ist eine deutliche Abweichung von der deutschen Syntax, die wahrscheinlich nicht auf den hohen Grad der Mündlichkeit zurückzuführen ist: „,[V]enn ich im fall noch nicht dort sollte sein...".

An anderer Stelle passt sich die Autorin mehr dem Schriftdeutschen an als zuvor. So setzt sie deutlich mehr Interpunktionszeichen und verwendet die normgerechte Schreibung der Konjunktion „dass“. Welche Faktoren diesen Wandel beeinflusst haben, kann nicht eindeutig konstatiert werden. Der Umzug mag die neuen portugiesischstämmigen Elemente erklären, lexikalische Entlehnungen waren hingegen seither Bestandteil des Vokabulars von Sprechern des Hunsrückischen (vgl. STEFFEN 2016: 147). Die stärkere Anpassung an das Schriftdeutsche ist vorerst ungeklärt. Eventuell orientierte sich das landschaftliche Hochdeutsch in Porto Alegre stärker am Hochdeutschen als das in Hamburgo Velho und die Autorin passte sich an die lokale Norm ihres neuen Zuhauses an. Die Beobachtungen zeigen, dass insbesondere die 
Codes „Hochdeutsch“, „landschaftliches Hochdeutsch“ und „Hunsrückisch“ oft kaum eindeutig voneinander $\mathrm{zu}$ trennen sind und dass diese Varietäten nebeneinander existieren sowie einander beeinflussen; dabei kann von verschiedenen Varianten des landschaftlichen Hochdeutsch ausgegangen werden, was wiederum nahelegt, dass dieses sich noch nicht als eigenständige Varietät stabilisiert hatte.

Die folgenden beiden Briefe wurden von einer älteren Person geschrieben. ${ }^{9}$ Allgemein ist ihr geschriebenes Deutsch dem Hochdeutschen deutlich näher als das der ersten Autorin. Dies kann durchaus an ihrem Alter liegen, wenn man davon ausgeht, dass sie zu einem früheren Zeitpunkt und an einem anderen Ort Deutsch in der Schule gelernt hat, an dem der Unterschied zwischen dem landschaftlichem Hochdeutsch und Hochdeutsch geringer war. Allerdings ist dies eine Vermutung.

Das normgerechtere Deutsch schlägt sich in folgenden Punkten nieder: Die Autorin setzt wesentlich mehr Interpunktionszeichen und schreibt Nomina durchgehend groß. Allgemein ist sie sehr viel konsequenter in der Anwendung von Regeln, die sie wahrscheinlich als lokale Norm in der Schule gelernt hat. Die ersten beiden Briefe sind aber nicht falsch oder schlechter, sie repräsentieren vielmehr eine andere lokale Norm. Im Vergleich zur ersten Autorin scheint die zweite Autorin sich beim Gebrauch ,ihrer“ Norm jedoch wesentlich sicherer zu fühlen.

Liebe Ida!

Nun warte ich schon so lange / auf einen Brief, aber es ko $\bar{m} \mathrm{t}$ kei $=/$ ner, hast du vergessen mir zu schrei $=$ / ben, oder hast du oder deine Schwes $=$ / tern keine Zeit? Ganz gewisz wohnt / Ihr doch jetzt schon in Eurem neuen / Hause, ich habe ja auch nicht an die / Finchen und Idalina zum Geburts / tage geschrieben, weil ich nicht die / Numer von Eurem neuen Haus / und den Namen von der Strasze / weisz, aber nun wünsche ich noch / heute recht viel Glück und Gottes / Segen für Finchen und Ida- / lina. [...] / Mir d ging es in letzter Zeit / nicht vom besten, die Reise / nach Hamburg war auch / nicht so gut als ich gehofft / hatte, habe überall Kranke / angetroffen, als ich zu Hause / kam, hat meine Schwägerin / Sophia schon hier auf mich / gewartet, wir wollten uns / in Hamburg treffen und / zusaen zuhause fahren, aber / als ich dort ankam da war sie / schon fort, da war sie längere / Zeit hier bei mir und war auch / krank geworden, aber jetzt ist / sie wieder in Porto Alegre / angestellt. Jetzt habe ich auch / mal wieder Unglück gehabt, / ich war von einer Spinne ge $=$ / bissen an der linken Hand u. / konnte so Tage nicht arbeiten / jetzt geht es schon wieder, aber / gut ist es noch nicht, doch hoffe / ich das es jetzt bald ganz gut / damit ich wieder besser arbei $i_{\text {ten }} /$ kann, ich habe doch wieder eine / Reise vor da musz ich doch was / verdienen, habe eine Bluse / zu stricken, [...] wenn die fer / tig ist und deine auch, dann / werde ich wenn Gott will wieder / nach Montenegro auf / besuch komen und die $\mathrm{Blu}_{=} /$sen hinbringen aber das /

\footnotetext{
${ }^{9}$ Verwandtschaftsbeziehung und Inhalte der Briefe lassen darauf schließen. In einem anderem hier nicht präsentierten Brief verweist die Autorin auf ihr hohes Alter, das sie von der Umsetzung ihrer Reisepläne abhält.
} 


\section{Woytowicz, L. Zerfall des Schriftdeutschen im Estado Novo?}

wird wohl noch ein paar Wochen / dauern, weil ja auch nähen / musz. Wie geht es sonst noch / noch in Montenegro? / Diesen Brief habe ich mit in Tante Lisette ihr Covert getan / damit er sicher anko $\bar{m}$ t weil ich die richtige Adresse von / Eurem neuen Haus nicht weisz / Viele herzliche Grüsze an Euch alle sendet Eure Tante Em a S.

[J.E.S., Canoas, 19/04/1936]

Anstelle der Graphemkombination „mm” schreibt die Autorin stets ein „m” und versieht es mit einem Strich, selbst bei ihrem eigenen Vornamen. Ebenso konsequent schreibt sie alle Pronomina der zweiten Person Plural groß, alle übrigen klein. Auch diese Regelmäßigkeiten sind wahrscheinlich auf einen erlernten Standard zurückzuführen, wobei das erste Phänomen auch pragmatische Gründe haben könnte. Ebenso wie die erste Autorin verwendet sie einen doppelten Bindestrich. Dieser wird jedoch immer tiefgestellt. Eine weitere Gemeinsamkeit ist die fehlende orthographische Unterscheidung zwischen ,dass“" und „das“.

Im Schriftbild der Verfasserin wird die Graphemkombination „,sz“ nicht zu einem „, $\aleph^{“}$ zusammengefasst; andere Autoren tun dies durchaus. An mancher Stelle verwendet sie auch „ss“ (,vergessen“, „Adresse“, „gebissen“). Es ist jedoch nicht festzustellen, ob die jeweiligen Graphemkombinationen unterschiedliche Laute umsetzen oder ob es sich um den gleichen Laut /s/ handelt. Letzteres ist wahrscheinlicher, es bleibt jedoch ungeklärt, wann die Autorin sich für welches Graphem entscheidet. Allerdings ist sie konsequent in ihrer Schreibung, da Wörter, die sie mehrmals benutzt, gleich geschrieben werden (,weisz“, „musz“).

Bei dem Nomen „Bluse“ könnte es aufgrund des portugiesischen Einflusses zu einer leichten Bedeutungsverschiebung gekommen sein. Ein Bedeutungswandel des Wortes ist jedoch auch ohne den Einfluss der Fremdsprache möglich. Eine Bluse im üblichen Sinne kann jedenfalls nicht gestrickt werden, da sie nicht aus Wolle besteht. Der deutschstämmige Ortsname „Hamburgo“ wird von der Verfasserin wieder eingedeutscht, die portugiesischstämmigen Ortsnamen bleiben unverändert. Diese Beispiele zeigen ein weiteres Mal, dass eine strikte Trennung der Varietäten bzw. Codes oft unmöglich ist.

Dem Inhalt des Briefs kann man entnehmen, dass die Autorin relativ viel verreist. Es ist daher davon auszugehen, dass sie regelmäßig mit verschiedenen Varietäten des Deutschen in Kontakt gekommen ist. Sie war also weniger isoliert als wahrscheinlich viele andere Sprecher des Hunsrückischen. Auch dies ist eine mögliche 
Erklärung für ihr stärkeres Bewusstsein für eine Norm und die daraus resultierende Normloyalität.

Der Abbau grammatikalischer Strukturen erfolgt nur selten. An einer Stelle fehlt ein Subjekt (,weil ja auch nähen musz“), außerdem enthält der Brief eine unvollständige Passivform (,war von einer Spinne gebissen“). Einmal wird der Genitiv durch ein Konstrukt aus Dativ und Possessivpronomen ersetzt (,,in Tante Lisette ihr Covert getan") und manchmal werden Präpositionen und Konjunktionen normabweichend verwendet (,nicht vom besten“, „so gut als ich gehofft hatte“, ,als ich zu Hause kam"). Hier gilt dasselbe wie in Bezug auf die ersten beiden Briefe. Mit dem Hochdeutschen als Maßstab handelt es sich dabei um Abweichungen von der Norm, was jedoch nicht bedeutet, dass es sich um Abweichungen von der lokalen Norm handelt. Weitere mögliche Erklärungen für diese Auffälligkeiten sind schlichte Nachlässigkeit (im Fall von fehlenden Wörtern), Interferenzen des Portugiesischen oder Mündlichkeit. Letztere liegt im landschaftlichen Hochdeutsch immer bis zu einem gewissen Grad vor, da dieses von einer rein mündlich verwendeten Varietät, dem Hunsrückischen, geprägt war. Ein weiterer Erklärungsversuch ist ein innerer typologischer Wandel, der in Sprachinseln schneller erfolgt (vgl. ROSENBERG 2003: 10; vgl. STEFFEN 2016: 147).

Der zweite Brief ist aus der Zeit, in der das Sprachverbot bereits galt. Die Autorin schreibt verspätet und auf Deutsch ,weil sie nicht weis, ob [die Leser] Brasilianische Briefe lesen [können]“. „Es scheint also so $\mathrm{zu}$ sein, dass das Sprachverbot sich offiziell auch auf den privatschriftlichen Bereich bezog, de facto aber in diesem Kontext nicht so streng durchgesetzt wurde“" (STEFFEN 2016: 151). Dieser Brief ist dem Schriftdeutschen sogar etwas näher als der erste. Eine mögliche Erklärung dafür ist der Umzug der Verfasserin nach „Betania“. Dabei handelt es sich höchstwahrscheinlich um eine Diakoniegemeinschaft in Curitiba (siehe Karte 2). Diese wurde erst im Jahr 1934 von Diakonissen aus Marburg gegründet (vgl. IRMANDADE EVANGÊLICA BETÂNIA 2017).

Ihr lieben Wernersleute!

Da ich heute gute Gelegenheit habe will / ich Euch ein paar Zeilen schreiben. / Zuerst wunsche ich Euch recht viel Glück und / Gottes Segen zum neuen Jahr, mit Gesundheit / und Freude. Ich hatte schon lange nichts mehr von / Euch gehört, ich habe auch schon lange keinen / Brief von Euch bekoen, man darf von hier aus / keine Deutschen Briefe mehr schreiben und weil / ich nicht weis ob Ihr Brasilianische Briefe lesen / könt deswegen habe ich nicht geschrieben. / Wie geht es Euch seid Ihr noch alle gesund? / wie es der Leopoldina wohnt sie noch in Roland? / Lebt die alte Frau Kaiser noch? / 
Woytowicz, L. Zerfall des Schriftdeutschen im Estado Novo?

Mir geht es gut habe auch imer viel zu nähen. / Bitte schreibt mir doch auch mal, recht viele grüse / an Euch alle von Tante /

Ema S.

[J.E.S., Betania, 04/01/1943]

Unübliche Formulierungen (,gute Gelegenheit habe“) und andere Auffälligkeiten (,weis“, ohne „sz“ wie im ersten Brief) können auch hier nicht eindeutig einer bestimmten Ursache zugeschrieben werden. Beim Vergleich der Briefe der beiden ersten Autorinnen wird jedoch deutlich, dass die verwendete Varietät stark vom Individuum und dessen Lebensumständen abhängt und dass wahrscheinlich keine einzige genormte Form des landschaftlichen Hochdeutsch besteht, sondern viele Varietäten des Schriftdeutschen vorliegen. Nicht nur das Hunsrückische wird als Dialektkontinuum betrachtet (ALTENHOFEN 1996: 27), auch das (geschriebene) landschaftliche Hochdeutsch ist anscheinend auf einem Kontinuum anzusiedeln. Die anderen Briefe, von denen drei weitere hier vorgestellt werden sollen, sprechen ebenfalls dafür. Nichtsdestotrotz kann man dem landschaftlichen Hochdeutsch einen übergeordneten Standard nicht vollkommen absprechen, da einzelne Phänomene sich in mehreren Briefen verschiedener Autoren aus unterschiedlichen Jahren nachweisen lassen. Darunter fallen Genitivverlust, der doppelte Bindestrich, die gleiche Verwendung und/oder Großschreibung von portugiesischen Lehnwörtern, Konjunktivformen des Verbs „tun“ in Verbindung mit dem Infinitiv und die fehlende orthographische Unterscheidung zwischen „das“ und „dass“. All diese Elemente finden sich auch in den folgenden beiden Briefen. Sie wurden der Einfachheit halber hervorgehoben.

Guerida Ida!

Im ersten Platz will ich Dir Glück / wünschen für dein Geburtstag / dem 24 ten, das du glücklich bist, und / gesund wirst, und noch viele Jahre lebst. / Und lade Euch alle freunlich ein für / dem 19 Setember dann ist die Alda / ihre Hochzeit, und nicht im Mai / wie die Leute gesagt haben. / [...] Das Geld können wir jetzt / un möchlich schicken, es geht jetzt mit dem / besten Willen nicht, denn wir haben / jetzt zuweil sonst alles zubezahlen, ich / hat mich schrechlich gekränk das wir das / Geld nicht schicken konten, aber wenn / die Alda verheiratet ist denn wollen / wir wieder 500 M. schicken, jetzt geht nich / [...] Alda hat heut schreklich geweint, die / D. Chiginha, die Diretora geht Morgen / fort von hier, geht nach Pirang dort ist / sie hin versetzt worden ver= / zeiht der Alda wenn sie noch / nicht geschriebn ${ }^{\text {hat }}$, die hat so viel / zuviel zutuhn, sie wird auch an / euch alle denken und schreiben, / überhabt an die Gote, die wo sie / so liebt, sie wird ein Bild mitschicken / und jedes mahl ist es schlecht ausgefallen / [...] Was wollt ich noch schreiben, ach / ja das die Iracema in São Leopoldo / war und hat examen gemacht, aber / sie ist nicht durch gekommen, ihr wißt / ja wie es immer ist, ihr habt doch / selbst gemeint sie soll nicht mehr / nach Montenegro kommen weil sie / nicht hören tät [...] / Alda war auch in Porto Alegre / hat den Kurs von Educação 
Fisica / gelernt hat ihr sie nicht in der / Zeitung gesehen mit ihre / Koleginen sie war in allen / Zeitungen, andere Leute könne / auch immer alles gut wissen / weil sie die Zeitungen leszen [...] / Viele Grüße von uns / alle an meiste von / L. H.

[L.H., Rolante, 12/08/1936]

Der Anfang des ersten Briefes der dritten Autorin zeigt, wie stark die einzelnen Varietäten ineinander übergreifen. Die Anrede kommt vom portugiesischen „querida“, wird aber orthographisch teilweise nach portugiesischen Regeln, teilweise nach hunsrückischer Aussprache umgesetzt. „Im ersten Platz“ ist eine wörtliche Übersetzung des Portugiesischen, die jedoch grammatikalisch und orthographisch dem Hochdeutschen entspricht.

Zwischen den beiden Briefen dieser Frau sind linguistisch kaum Unterschiede auszumachen. Auf einem möglichen Kontinuum des landschaftlichen Hochdeutsch liegt die von ihr verwendete Varietät vermutlich zwischen den Varietäten der beiden anderen. Einerseits schreibt sie wie die zweite Autorin Nomina meist groß, andererseits ist sie ebenso wie die erste inkonsequent bei der Schreibung einiger Wörter (,schrechlich“ vs. „schreklich“, ,nich“ vs. ,nicht). Der Grad an Mündlichkeit ist hoch. Im Gegensatz zur ersten Autorin setzt sie mehr Interpunktionszeichen und schreibt weniger redundant; Orthographie und einige Formulierungen sind mündlich markiert (,un möchlich“, „wollt“, ,freunlich“, „Was wollt ich noch schreiben, ach..."). Selten kommt es zu Vokalverschiebungen (,überhabt“). Einige sprachliche Besonderheiten sprechen sowohl für allgemeine grammatikalische Verluste als auch für Mündlichkeit, wie z.B. fehlende Verbendungen („könne“) oder Kasusverlust („für dein Geburtstag“, „,ein Monat“", „so ein Hals“").

Liebe Mutter und Geschwiester!

Nun ist es schon wieder ein Monat / Her das ich euch verlassen hab, / Wie geht es euch? Seit ihr noch alle / gesund, uns get es nicht von aller- / beste, wir hatten alle so ein Hals / das wir nächst nicht schlucken konten. / Jetzt ist es schon wieder etwas besser. / Nun wünsche ich euch fröhliche Pfingsten / Iracema, hat auch geschriben, es wäre / ganz schön dort, die Stadt und die / Alda sein Haus, aber wie es aus / seht will sie wieder kommen. / Die Alda macht wieder den nächsten / Monat ein curso, denn will die Lena / wieder mitkommen. / Und die Reise zu Hause war sehr schnell / um halb elf Uhr war ich schon zu / Hause gewessen, das was alles vermoft / vergraut, man seht gleich wenn man / nicht zu Hause ist. Mit weil Glück / und Gesundheit vershliese ich mein schreiben / L.

[L.H., Rolante, 28/05/1939]

Die dritte Autorin unterscheidet ebenso wie die zweite nicht zwischen „nach Hause“ und ,zu Hause“, sowohl die Orts- als auch die Richtungsangabe wird mit „zu Hause“ 
ausgedrückt; eine weitere Gemeinsamkeit ist der Ausdruck ,nicht von allerbeste [gehen]“. In beiden Fällen kann davon ausgegangen werden, dass es sich auch hier um einen überregionalen Standard handelt, d.h. um ein distinktives Merkmal des landschaftlichen Hochdeutsch im Vergleich zum Hochdeutschen. Der zweite Brief dieser Autorin zeigt außerdem, dass lexikalische Entlehnungen des Portugiesischen durchaus mit deutschen Morphemen kombiniert werden. Das Partizip „vermoft“ geht auf das portugiesische Wort für Schimmel (= mofo) zurück. Anstatt diese Fälle als reine Interferenzen abzutun, kann man sie auch als Indikator für Kreativität und Wortschöpfungsprozesse betrachten. Gleiches gilt für alternative Verwendung von Präfixen (,vershliese“).

Der letzte Brief stammt von einer Autorin, die sich sehr weit von der Mutterkolonie entfernt hat (siehe Karte 2). Dies ist eine mögliche Erklärung dafür, dass ihre Normloyalität so gering ist, jedoch sicherlich nicht die einzige, da die erste Autorin, deren geschriebenes Deutsch sehr vergleichbar ist, im näheren Umfeld von São Leopoldo wohnte.

Teure Freundin Ida und Geschvister

Mit freude habe Ich deine Karte erhalten; vofür / Sage Ich meinen Innigsten Danck aus, jedesmal / ven Ich vas von Euch zu hören bekome; denke Ich / an unsere schöne geselchaft dort zusammen, hier / giebt es nicht solche siten, hier ist es mit / freu ${ }^{\mathrm{e}}$ nchaft blosse sehr interessant die Mädeln / sietsen hier alle Tagen auf der Strasse spatzieren / ja ver aber so vie Ich von Motags bis sonabends / im Geschaft stehen muss, der kan nicht mit / halten, da habe Ich 3 oder 4 Mädchen Idaliener / das sind meine treueste freu endinnen, wir hand- / arbeiten des Abends zusamen, bald bei eine den / bei der Andere dies ist hier mein einziges vergnigen. / Wie gehet es Euhre gute Mutter, hoffentlich / gut, habe auch schon verlangen nach Euch alle / aber Ich veis nicht vie alles auf der Veld als / ist, mir gehet es gut, nur mit der Gesundheit / nicht fast so wie immer, wen Gott vil verde Ich / zu Veinachten spatzieren komen, oft veine / Ich habe verlangen, und zugleich pech in liebe / aber auf der andere seide, gehet es gut, darum / muss man dencken vas Gott tut ist. Wohlgetan. / Nun muss Ich meinen schreiben schliessen / und Euch Alle herzlich Grüssen, Euhre Freundin / in der ferne. /H.

[H., Santa Rosa, 12/03/1939]

Es scheint, dass die Schreiberin dieses Briefes im Gegensatz zur zweiten Autorin nach ihrem Umzug nach Santa Rosa weniger Kontakt mit Hunsrückischsprechern und (landschaftlichem) Hochdeutsch hatte. Zum einen erwähnt sie, dass ihre einzigen Freundinnen „Idaliener“ seien, zum anderen beinhaltet der Brief die bereits bei den anderen Briefen diskutierten Elemente des mündlichen Sprachgebrauchs. Diese sind jedoch stärker ausgeprägt, was dafür spricht, dass sich die Autorin aufgrund ihrer sozialen und geographischen Abgeschiedenheit das in der Schule erlernte 


\section{Woytowicz, L. Zerfall des Schriftdeutschen im Estado Novo?}

landschaftliche Hochdeutsch wenig benutzt, Portugiesisch kaum spricht und sich daher zwangsläufig beim Schreiben noch stärker an ihrer Muttersprache Hunsrückisch orientiert als die meisten anderen Schreiber. Mit dem Sprachverbot wurde der Kontakt mit den deutschen Varietäten wahrscheinlich darüber hinaus eingeschränkt.

Beim Vergleich aller Briefe fällt auf, dass insbesondere bei der graphemischen Umsetzung von Sibilanten eine große Varianz vorliegt, sodass man vom Schriftbild nicht immer eindeutig auf die Aussprache schließen kann:

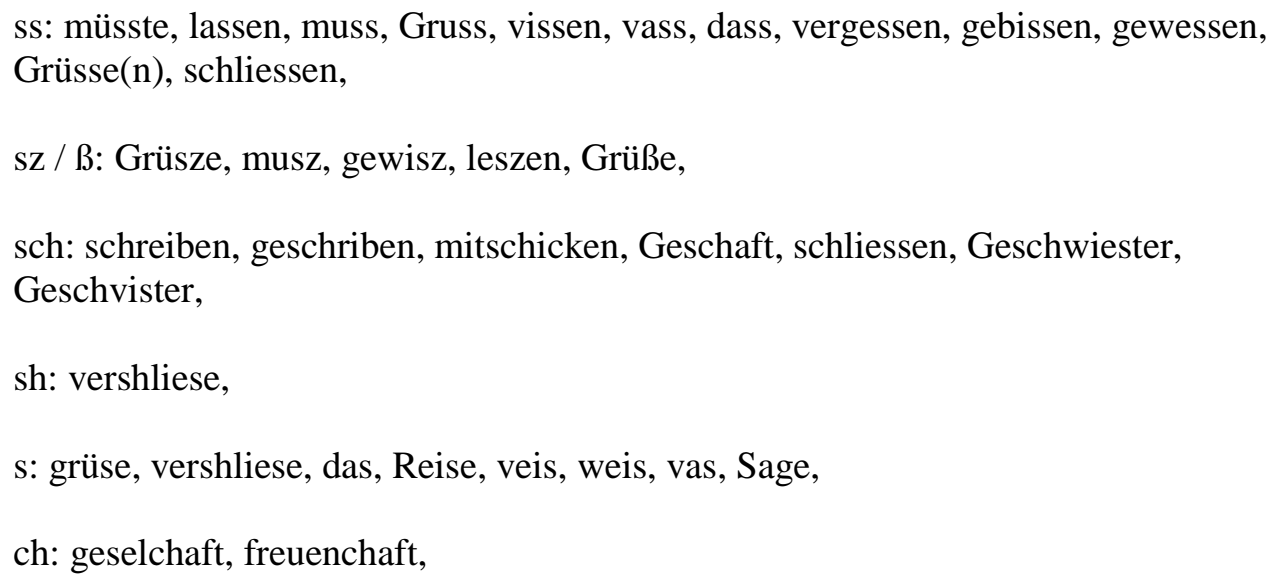

Diese Beobachtung legt nahe, dass die Umsetzung von Sibilanten im landschaftlichen Hochdeutsch eher zufällig erfolgte. Aus diesem Grunde entschieden die Autoren wahrscheinlich oft individuell über die Schreibung des jeweiligen Wortes.

Zur klareren Abgrenzung eines übergeordneten lokalen Standards des Schriftdeutschen unter den Nachfahren der deutschen Einwanderer, müsste man das Deutsch der Publikationen aus der Zeit vor dem Estado Novo mit dem der Privatbriefe vergleichen. Man sollte jedoch nicht vergessen, dass es nicht die hauptsächliche Intention der Autoren war, normgerecht zu schreiben; in erster Linie wollten sie kommunizieren. Eine passive Kompetenz des Schriftdeutschen lag durchaus vor: So bezog Ida Werner beispielsweise per Post Horoskope aus Deutschland. Außerdem ist aktuellen Studien zufolge die Lesekompetenz derjenigen, deren Elterngeneration noch Deutsch in der Schule gelernt hat, beim Deutschen höher als beim Portugiesischen; in den nachfolgenden Generationen ist aufgrund des Dachsprachenwechsels das Gegenteil der Fall (vgl. AltenhOFEN 2016: 122).

Die Analyse der Briefe hat gezeigt, dass in allen Generationen - d.h. auch in der Phase der Zweisprachigkeit, in der Sprecher des Hunsrückischen Deutsch in der Schule lernten - die meisten bis zu einem gewissen Grad so schrieben, wie sie sprachen. 
Mündlichkeit im Schriftdeutsch dieser Sprechergruppe ist daher an und für sich kein Indiz für eine fehlende Sprachkompetenz im Hochdeutschen. Das Verschwimmen der Grenzen zwischen den Varietäten, insbesondere zwischen landschaftlichem Hochdeutsch und Hunsrückisch, wurde in der Analyse mehrfach aufgezeigt. Diese beiden Codes wurden in der geschriebenen Sprache nie strikt voneinander getrennt. Da den Autoren ihre Muttersprache Hunsrückisch immer näher war als andere Varietäten, war es auch in der geschriebenen Sprache stets präsent, selbst wenn der Grad der Präsenz variierte und sicherlich nicht immer vom Verfasser gewollt war.

Das Ausmaß mündlicher Elemente im Schriftdeutschen war individuell vom Autor abhängig; das, was der jeweilige Autor als Hochdeutsch empfand und anstrebte, wurde oft auch durch den Wohnort bestimmt. ${ }^{10}$ Der Wohnort war ausschlaggebend dafür, welche Varietät des Deutschen der Autor in der Schule lernte, wie häufig er diese Varietät benutzte und wie häufig er Kontakt zu Menschen hatte, die die gleichen bzw. andere Varietäten und Sprachen beherrschten. Eine Änderung des Wohnorts schlug sich daher oft im Sprachgebrauch der Betroffenen nieder.

Gerade aufgrund der starken individuell markierten Ausprägung des landschaftlichen Hochdeutsch sollte es ebenso wie das Hunsrückische auf einem Kontinuum angesiedelt werden. Einige Regelmäßigkeiten lassen darauf schließen, dass es durchaus einen überregionalen schriftlichen Standard gab, jedoch war dieser längst nicht so etabliert wie die Schriftform des Portugiesischen bzw. des Hochdeutschen (in Deutschland).

\section{Abschlussbemerkungen}

Es scheint, als ob Faktoren wie geografische und soziale Isolation, Binnenmigration und die Präsenz mehrerer deutscher Varietäten in verschiedenen Regionen die Etablierung einer einzigen deutschen Schriftnorm unmöglich machten. So blieb das Hochdeutsche bis zu seinem Verbot die (Pseudo-)Dachsprache der deutschen Varietäten in Brasilien, sein Fundament war jedoch relativ schwach. Schon vor dem Estado Novo zerfiel es in mehrere regional und individuell geprägte Varietäten, was anhand der Briefe deutlich zu

\footnotetext{
${ }^{10}$ Andere mögliche Faktoren, auf die in diesem Artikel nicht eingegangen wird, sind beispielsweise der Bildungsgrad, das Alter oder das Geschlecht der Person.
} 


\section{Woytowicz, L. Zerfall des Schriftdeutschen im Estado Novo?}

erkennen ist. Darüber hinaus wurde es im Alltag derjenigen, die nicht (mehr) zur Schule gingen, aufgrund seiner diaphasischen Abgrenzung zum Hunsrückischen aktiv kaum benutzt; es diente den Verfassern von Briefen als Orientierung und wurde durch Zeitungen und andere Publikationen rezipiert. Abgesehen davon wurde es selten verwendet, da viele Sprecher des Hunsrückischen in ihrem Alltag wenig aufschrieben. Mit dem Estado Novo fand jedoch auch jegliche passive Nutzung des landschaftlichen Hochdeutsch ein jähes Ende.

Das Hunsrückische genoss im Gegensatz zum Hochdeutschen kaum Prestige; es war stets die Mutter- aber nie die Bildungssprache seiner Sprecher. ${ }^{11}$ Aufgrund dessen entwickelte sich nur eine kleine, private Schreibtradition des Hunsrückischen. Erst viel später wurde der Versuch unternommen, diese Varietät auch schriftlich zu standardisieren. Daher war die einzige überregionale genormte Schriftsprache stets das Portugiesische. Die meisten Hunsrückischsprecher hatten jedoch nie gelernt, Portugiesisch normgerecht zu schreiben. Aufgrund des Sprachverbots durften sie nicht einmal mehr eine Schriftnorm verwenden, bei der sie wenigstens das Gefühl hatten, sie zu beherrschen. Dieser Punkt hat sicherlich entscheidend dazu beigetragen, dass die Post-Estado-Novo-Generation wieder bewusst begann, Texte auf Hunsrückisch zu verfassen (vgl. PUPP SPINASSÉ 2016: 88); die Etablierung eines einzigen landschaftlichen Hochdeutsch war spätestens durch das Sprachverbot endgültig verhindert worden.

Schon weit vor dem Estado Novo kam es zu einer ersten Phase der von RosenberG (2003: 14) beschriebenen Norminstabilität, die zu Mischvarietäten und Sprachmischung führte. Hunsrückisch selbst ist eine solche Mischvarietät. Da die deutschen Einwanderer der ersten Generation unterschiedliche Varietäten sprachen und mit Portugiesischsprechern in Kontakt kamen, lösten sich schon ab 1824 sprachliche Gruppengrenzen auf (vgl. ebd.). Der Estado Novo stellt eine zweite Phase von Norminstabilität dar, und das gleich auf mehreren Ebenen: Der Staat wurde unter Vargas allgemein umstrukturiert, Systemgegner wurde verfolgt, während in Europa der Zweite Weltkrieg ausbrach. Der schlechte Ruf der Deutschen wirkte sich negativ auf den Ruf der Sprecher deutscher Varietäten in Brasilien aus (vgl. PUPP SPINASSÉ 2016: 85), die außerdem aufgrund des Sprachverbots staatliche Repressionen fürchten

${ }^{11}$ An diesem Umstand hat sich auch bis heute kaum etwas geändert. 
mussten. Offensichtlich war diese Phase in mehrerlei Hinsicht eine Zeit des Umbruchs, in der den Hunsrückischsprechern die einzige Norm, die sie in der Schriftsprache teilweise entwickelt hatten, genommen wurde. Eine Schriftnorm des Hunsrückischen gab es nicht, Portugiesisch beherrschten sie nur teilweise. Auch deswegen konnte das Hochdeutsche als Dachsprache so leicht verdrängt werden und an seinen Platz rückte das Portugiesische anstatt einer einzigen geschriebenen Norm des landschaftlichen Hochdeutsch, die sich ggf. entwickelt hätte. Zugegebenermaßen wäre diese sowohl vom Portugiesischen als auch vom Hunsrückischen beeinflusst gewesen, aber genau das hat das landschaftliche Hochdeutsch stets vom Hochdeutschen abgegrenzt.

Bei der Frage, ob der Dachsprachenwechsel die Redialektalisierung förderte oder ob letztere den Dachsprachenwechsel vorantrieb, handelt es sich wohl um ein klassisches Henne-Ei-Dilemma: Hunsrückisch diente seinen Sprechern in Zeiten großer Norminstabilität und schriftsprachlicher Alternativlosigkeit als Refugium, das ihnen im Gegensatz zum Portugiesischen Kontinuität, Identität und Sicherheit gab; dies hatte wiederum einen beschleunigten Niedergang des Hochdeutschen zur Folge, der vom Regime durchaus gewollt war und der innerhalb weniger Jahre Hunsrückischsprechern keine andere Wahl mehr ließ, als auf Portugiesisch zu schreiben, da sie des Deutschen nicht mehr mächtig waren und ihre Muttersprache über keine schriftliche Norm verfügte.

Anzeichen für eine abnehmende Normloyalität wurden durch STEFFEN bereits in Briefen nachgewiesen, die lange vor dem Estado Novo verfasst wurden:

Der Sprachwechsel im Bereich der Privatschriftlichkeit in Südbrasilien war demnach eher ein kontinuierlicher, aber sehr langsam ablaufender Prozess, der durch das allmähliche Ein- und Vordringen des Portugiesischen bei gleichzeitigem Abbau des Hochdeutschen geprägt war und der bereits um die Jahrhundertwende [...] begonnen hatte. (STEFFEN 2016: $154 \mathrm{f}$.)

Der Dachsprachenwechsel kann daher nicht ausschließlich mit der Sprachpolitik unter Vargas erklärt werden. Ein Abbau der Hochdeutschen ist auch in den hier präsentierten Briefen erkennbar; die Frage ist, ob es sich dabei um einen adäquaten Maßstab für die Einordnung des landschaftliche Hochdeutsch handelt. Denn in keiner Phase entsprach das geschriebene Deutsch der Hunsrückischsprecher dem geschriebenen Hochdeutsch. Außerdem scheint die Nähe bzw. Distanz zum Hochdeutschen viel mehr vom Individuum selbst als von anderen Faktoren anhängig zu sein. Fest steht, dass das landschaftliche Hochdeutsch in Brasilien mit seinen letzten Schreibern ausstarb, da für 
Woytowicz, L. Zerfall des Schriftdeutschen im Estado Novo?

die nachfolgenden Generationen das Erlernen einer schriftlichen deutschen Norm unmöglich war und mangels Anwendungsmöglichkeit überflüssig erschien. Wir wissen nicht, wie sich das landschaftliche Hochdeutsch entwickelt und ob es sich als eigenständige Varietät etabliert hätte, wenn es nicht konsequent verboten worden wäre. Ebenso wenig wissen wir, ob das Hunsrückische unter anderen Umständen schon zu einem früheren Zeitpunkt verschriftlicht worden wäre.

\section{Literaturverzeichnis}

AltenhofEn, Cléo V. Hunsrückisch in Rio Grande do Sul. Ein Beitrag zur Beschreibung einer deutschbrasilianischen Dialektvarietät im Kontakt mit dem Portugiesischen. Dissertation. Stuttgart: Steiner, 1996.

Altenhofen, Cléo V. O "Território de uma Língua": Ocupação do Espaço Pluridimensional por Variedades em Contato na Bacia do Prata. In: DA RoSA NIEVES FERNÁNDEZ, Ana Lourdes; MOZZILlo, Izabella; NilSe SCHNEIDER, Maria; CoRTAZZO, Uruguay (Hg.): Línguas em contato: onde estão as fronteiras? Pelotas. Editora da UFPel, 2014, 69-104.

Altenhofen, Cléo V. Standard und Substandard bei den Hunsrückern in Brasilien: Variation und Dachsprachenwechsel des Deutschen im Kontakt mit dem Portugiesischen. In: BÜRING, Daniel; LENZ, Alexandra N.; RITT, Nikolaus (Hg.): German Abroad: Perspektiven der Variationslinguistik, Sprachkontakt- und Mehrsprachigkeitsforschung. Göttingen. V\&R unipress, 2016, 103-129.

Altenhofen, Cléo V.; Frey, Jaqueline; KäFer, Maria Lidiani; Klassmann, Mário S.; NeUmanN, Gerson R.; PUPP SPINASSÉ, Karen. "Fundamentos para uma escrita do hunsrückisch falado no sul do Brasil". In: Revista Contingentia 2, 2/2007, 73-87. http://seer.ufrgs.br/index.php/contingentia/article/view/3867/2166 (21/04/2017).

BußManN, Hadumod. Lexikon der Sprachwissenschaft (4. Auflage). Stuttgart. Alfred Kröner Verlag, 2008.

BRANFORD, W.; Claughton, J.S. Mutual lexical borrowings among some languages of southern Africa: Xhosa, Afrikaans and English. In: MesthriE; R. (Hg.): Language and Social History, Studies in South African Sociolinguistics (1. Auflage). Kapstadt und Johannesburg: David Philip Publishers, 1995, 209-221.

DUDENREDAKTION. ,Schriftdeutsch“ auf Duden online. http://www.duden.de/rechtschreibung/ Schriftdeutsch (25/06/2917).

Fleischer, Wolfgang; Helbig, Gerhard; LerCHNER, Gotthard (Hg.). Kleine Ezyklopädie Deutsche Sprache. Frankfurt. Peter Lang, 2001.

GoOGLE (Hg.). Google Maps. Screenshot - Meine Karten. Absender. https://www.google.com/maps/d/edit?hl=de\&authuser=0\&mid=1D3YXmlEut7x1LqDeH5s 91dFPynU\&ll=-29.747679864445068\%2C-51.2483334144531\&z=10 (22/04/2017).

IRMANDADE EVANGÊLICA BETÂNIA. Flyer. Curitiba. http://diakonissenmutterhaushebron.de/sites/diakonissenmutterhaus.extrafolge.de/files/dialog/-downloads/Flyer_Brasilien-Betania.pdf (10/04/2017).

PUPP SPINASSÉ, Karen. Das brasilianische Hunsrückische: soziolinguistische Aspekte einer durch Sprachkontakt geprägten Minderheitensprache. In: BÜRING, Daniel; LENZ, Alexandra N.; RiTT, Nikolaus (Hg.): German Abroad: Perspektiven der Variationslinguistik, Sprachkontakt- und Mehrsprachigkeitsforschung. Göttingen. V\&R unipress, 2016, 81-102. 
Woytowicz, L. Zerfall des Schriftdeutschen im Estado Novo?

ROSENBERG, Peter. Vergleichende Sprachinselforschung: Sprachwandel in deutschen Sprachinseln in Russland und Brasilien. In: Linguistik online 13, 1/2003. http://www.linguistik-online.org/13_01/rosenberg.html (10/04/2017).

STEFFEN, Joachim. Einblicke in einen Sprachwechsel in Zeitlupe: Phasen des deutschportugiesischen Sprachkontakts in Südbrasilien in Briefen aus zwei Jahrhunderten. In: BÜRING, Daniel; LENZ, Alexandra N.; RITT, Nikolaus (Hg.): German Abroad: Perspektiven der Variationslinguistik, Sprachkontakt- und Mehrsprachigkeitsforschung. Göttingen. V\&R unipress, 2016, 131-157.

Recebido: 03 mai. 2017

Aceito: 12 jul. 2017 